Horizons philosophiques

\title{
Philosophie et technique autour de l'université
}

\section{Takuji Iwano}

Volume 5, numéro 2, printemps 1995

Le philosophe et l'état

URI : https://id.erudit.org/iderudit/800980ar

DOI : https://doi.org/10.7202/800980ar

Aller au sommaire du numéro

\section{Éditeur(s)}

Collège Édouard-Montpetit

\section{ISSN}

1181-9227 (imprimé)

1920-2954 (numérique)

Découvrir la revue

\section{Citer cet article}

Iwano, T. (1995). Philosophie et technique autour de l'université. Horizons philosophiques, 5(2), 49-73. https://doi.org/10.7202/800980ar d'utilisation que vous pouvez consulter en ligne.

https://apropos.erudit.org/fr/usagers/politique-dutilisation/ 


\section{PHILOSOPHIE ET TECHNIQUE AUTOUR DE L'UNIVERSITÉ}

Dans notre société industrialisée, la technique se renouvelle très rapidement. Face à cette transformation, qu'est-ce que la philosophie doit faire? Quelle attitude le philosophe doit-il prendre?

I) Dans La Métaphysique, Aristote parle de la hiérarchie des sens : "Tous les hommes désirent naturellement savoir; ce qui le montre, c'est le plaisir causé par les sensations, car, en dehors même de leur utilité, elles nous plaisent par ellesmêmes, et, plus que toutes les autres, les sensations visuelles. En effet, non seulement pour agir, mais même lorsque nous ne nous proposons aucune action, nous préférons, pour ainsi dire, la vue à tout le reste. La cause en est que la vue est, de tous nos sens, celui qui nous fait acquérir le plus de connaissances et nous découvre une foule de différences ${ }^{1}$ ". Ce primat de la vue sur d'autres sens et sa proximité avec la connaissance influencent, au-delà de la théorie de la sensibilité chez Aristote, l'orientation de sa philosophie tout entière. La hiérarchie des sens se tourne à l'ordre scientifique. Parce que la qewria - qui se lie étymologiquement à la vue - tient le premier rang dans le système scientifique d'Aristote. La supériorité de ce savoir théorétique (théorique ou contemplatif) sur celui de production

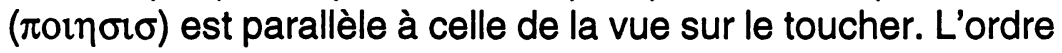
sensible sous-tend ainsi la hiérarchie scientifique. Or nous nous demandons comment se constitue l'ordre scientifique. Je schématise grossièrement la division des connaissances dans

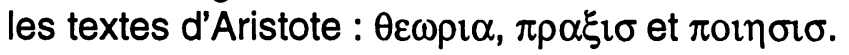

(1) $\theta \varepsilon \omega \rho \iota \alpha$. La supériorité d'une science se détermine selon le degré de sa proximité avec l'origine(arch). Si la $\theta \varepsilon \omega \rho \imath \alpha$, issue de la vue, a une primauté sur d'autres connaissances, c'est que ce savoir est le plus proche de l'origine, c'est-à-dire le principe(arch). Aristote écrit : “(...) sous la dénomination de

1. Aristote, La Métaphysique, t. 1, tr. fr. par J. Tricot, Vrin, 1986, 980a, p.2. 
sagesse, chacun entend communément ce qui traite des premières causes et des premiers principes; aussi, (...), juge-ton d'ordinaire l'homme d'expérience supérieur à l'homme qui a simplement une sensation quelconque, l'homme d'art supérieur à l'homme d'expérience, l'architecte au manœuvre, et les sciences théorétiques aux sciences pratiques. II est donc évident, dès maintenant, que la Sagesse est une science qui a pour objet certaines causes et certains principes" $"$. Par conséquent, Aristote privilégie trois philosophies ou sciences théorétiques qui recherchent l'être : (i) la physique, qui a pour objet d'étude l'être de la nature; (ii) la mathématique, qui étudie l'être abstrait, à savoir point, ligne, espace, etc.; (iii) la première philosophie, c'est à dire la métaphysique, qui explique le principe ou la cause de l'être en tant qu'être. Parmi ces trois ontosciences, la première philosophie atteint au sommet scientifique, car elle poursuit ce qui est le plus originel : "la science maîtresse», "qui est supérieure à toute science subordonnée», est "une science qui spécule sur les premiers principes et les premières causes ${ }^{3}$. La valeur d'une science relève de son rapport avec l'originel. Les sciences théorétiques qui s'appuient sur la sensibilité visuelle sont les plus proches de l'originel : le premier principe et la première cause. C'est pourquoi elles sont supérieures aux sciences pratiques et aux techniques.

(2) $\pi \rho \alpha \xi i \sigma$. Dans le système aristotélicien des connaissances, on voit un domaine des philosophies, les sciences pratiques, qui s'occupent des actions, des relations humaines et de la communauté. La politique et l'éthique en font partie. Leur étude ne consiste pas à rechercher ce que les choses sont, mais à penser comment on s'approche du bien, dubonheur ou de la justice. Il s'ensuit de là que leur connaissance "prudence» ( $\phi \rho o v \eta \sigma i \sigma)$ n'a pas une valeur théorique mais pratique. Toutefois la poursuite pratique du bonheur ramène, enfin, à une réaffirmation de la $\theta \varepsilon \omega p r \alpha$. L'idéal de la vie éthique réside dans la vie contemplative: «(...) pourl'être vivant, une fois

2. Ibid., 981b-982a, p.10-12.

3. Ibid., 982b, p.15-16, souligné par nous. 
qu'on lui a ôté l'action et à plus forte raison la production, que lui laisse-t-on d'autre que la contemplation? Par conséquent, l'activité de Dieu, qui en félicité surpasse toutes les autres, ne saurait être que théorétique. Et par suite, de toutes les activités humaines celle qui est la plus apparentée à l'activité divine sera aussi la plus grande source de bonheur ${ }^{4}$ ". Voilà une relation

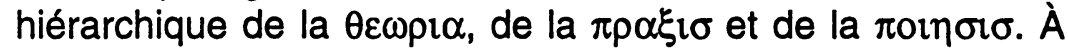
mesure que l'on s'éloigne de la production et de l'action, on participe à la vie théorétique originelle du dieu. Il est indéniable que la praxis est inférieure à la qewria. Mais puisque la politique et l'éthique portent le nom de "philosophie" ou de "science", elles sont plus estimées que ce qui s'appelle simplement $\tau \varepsilon \chi v \eta$.

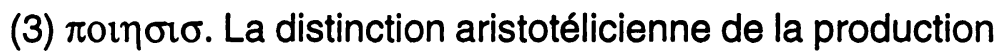
et de l'action conduit à réduire à la première la technique ( $\varepsilon \varepsilon \vee \eta):$ :...) puisque production et action sont quelque chose de différent, il faut nécessairement que l'art(tecnh) relève de la production et non de l'action ${ }^{5}$ ". La technique s'entend enfin au sens de "l'art de produire". Dans Éthique à Nicomaque, il insiste sur "une identité entre art et disposition à produire accompagnée de règle exacte ${ }^{6} \%$. En principe, ni $\theta \varepsilon \omega p \iota \alpha \mathrm{ni}$ $\pi \rho \alpha \xi i \sigma$ ne se nomment $\tau \varepsilon \chi \nu \eta$. Ce nom est réservé à la poihsis. On compte parmi les techniques la poétique, l'architectonique, la rhétorique, le tissage, etc. Et ce qui est important, c'est qu'aucune d'elles ne mérite de s'appeler "philosophie» ou "science». Par conséquent, le savoir technique est en dernier dans la hiérarchie aristotélicienne.

II en résulte que la connaissance philosophique, soit théorique soit pratique, est supérieure à la connaissance technique et que les techniques en tant qu'arts de production sont écartées du domaine philosophique. Aristote affirme : «[la première philosophie] n'est pas une science productive ${ }^{7}$ ". La science dont le statut est assuré par sa proximité avec l'origine, le premier principe ou la première cause laisse tomber la

\footnotetext{
4. Id., Ethique à Nicomaque, tr. fr. par J. Tricot, Vrin, 1983, 1178b, p.518.

5. Ibid., 1140b, p.285.

6. Ibid., 1140a, p.283.

7. Aristote, La Métaphysique, op. cit., 982b, p.16, modifié.
} 
technique. Autrement dit, la philosophie ne s'approprie le savoir originel que si elle dépasse le savoir technique. La science nommée "philosophie" se rapporte essentiellement à ce qui est non-technicisable ou sur-technicisable. La recherche de l'origine s'achève à mesure que la technique ou le technicisable s'écartent de lui. Le rapport de la philosophie et de la technique dépend ainsi de l'ordre hiérarchique : $\theta \varepsilon \omega \rho \imath \alpha>\pi \rho \alpha \xi 1 \sigma>$

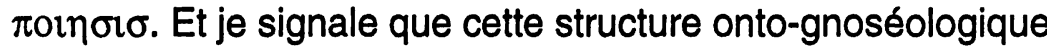
exerce une forte influence sur l'histoire des idées postaristotéliciennes. Les philosophies, les sciences, les idéologies et les systèmes pédagogiques de l'antiquité et du moyen âge ont été plus ou moins dominés par la suprématie de la $\theta \varepsilon \omega \rho t \alpha$ et le mépris de la technique. Cela s'explique, par exemple, par le fait que la vie contemplative l'a emporté sur l'engagement politique ou par le fait que l'on a plus considéré l'étude théorique dans l'université que l'activité productive. Les "arts libéraux" du moyen âge peuvent se définir, en un sens, comme des techniques non-technicisables, c'est-à-dire des techniques libres des techniques en tant que "arts de production".

II) La primauté de la philosophie sur le savoir technique se trouve également dans l'époque moderne. Je prends l'exemple de Kant. Dans le chapitre intitulé "Architectonique de la raison pure" de la Critique de la raison pure, Kant dit : "Entre toutes les sciences rationnelles (a priori), il n'y a donc que la mathématique qui puisse être apprise, mais jamais la philosophie (à moins que ce ne soit historiquement) : en ce qui concerne la raison, on ne peut apprendre tout au plus qu'à philosopher ${ }^{8}$ ". Ce philosophe allemand pense que la connaissance de la raison est divisée en deux catégories : "connaissance par concepts" et "connaissance par la construction des concepts". À la dernière appartient la mathématique, car la source de ses connaissances consiste dans les principes irrécusables de la raison. Comme cette science commence par ces principes absolument certains, on peut accéder, au moyen des raisonne-

8. Emmanuel Kant, "Critique de la Raison pure», tr. fr. par J.-L. Delamarre et François Marty, in Oeuvres philosophiques I, Gallimard, pléiade, 1980, B866, p.1388. 
ments déductifs, aux vraies connaissances rationnelles. Mais la philosophie en est différente, parce que l'on ne peut pas apprendre la philosophie, on ne peut apprendre qu'à philosopher. Qu'est-ce que cela signifie? Notons cependant une réserve de Kant : «à moins que ce ne soit historiquement". Il est possible d'apprendre la philosophie si elle s'interprète comme une connaissance historique, c'est-à-dire cognitio ex datis . Certainement, on peut apprendre, mémoriser et même réciter, par exemple, la philosophie de Wolff en tant que connaissance historique. Cependant cette sorte de travail n'est pas philosophique au sens objectif mais seulement une assimilation de la description historique de la philosophie. Dans la connaissance philosophique définie comme "connaissance par concepts", on voit donc une scission : connaissance historique et connaissance rationnelle. Alors que la philosophie au sens objectif est cette dernière connaissance, le philosophe est obligé de partir de la connaissance historique sans jamais en sortir. Quelque effort qu'il fasse, il n'accède pas à la philosophie au sens objectif. Elle n'est pas l'objet d'enseignement ou d'apprentissage. Kant dit: «(...) le système de toute connaissance philosophique est la philosophie. On doit la prendre objectivement, si l'on entend par là le modèle qui permet d'apprécier toutes les tentatives de philosopher, appréciation qui doit servir à juger toute philosophie subjective, dont l'édifice est souvent si divers et si changeant. De cette manière la philosophie est la simple idée d'une science possible, qui n'est donné nulle part in concreto, mais dont on cherche à se rapprocher par différentes voies, jusqu'à ce que l'on ait découvert l'unique sentier qui y conduit, mais que faisait dévier la sensibilité, et que l'on réussisse, autant qu'il est permis à des hommes, à rendre la copie, jusqu'à présent manquée, semblable au modèle ${ }^{9}$." La philosophie est un archétype. Kant définit la relation de la philosophie objective à notre philosophie comme étant celle de l'origine à une copie. La philosophie réelle n'est qu'une imitation de la philosophie en tant qu'origine. Comme 
notre philosophie n'échappe pas à la connaissance historique, nous ne pouvons que copier le prototype idéal. La philosophie est essentiellement inaccessible ou non appropriable comme une «idée transcendantale».

Kantessaie, d'autre part, d'exclure du concept de philosophie originelle le facteur technique : «Le mathématicien, le physicien, le logicien, quelque éclatant succès que puissent avoir les uns en général dans la connaissance rationnelle et les autres en particulier dans la connaissance philosophique, ne sont toutefois que des artistes de la raison (Vernunftkünstler). Il y a encore un maître dans l'idéal, qui les emploie tous, et se sert d'eux comme d'instruments pour avancer les fins essentielles de la raison humaine. C'est celui-là seul que nous devrions appeler le philosophe ${ }^{10}$ »; "(...) la philosophie est la science du rapport de toute connaissance aux fins essentielles de la raison humaine (teleologia rationis humanae), et le philosophe n'est pas un artiste de la raison (Vernunftkünstler), mais le législateur de la raison humaine ${ }^{11}$ ". Kant affirme icila supériorité de la philosophie idéale sur d'autres sciences. À l'en croire, la mathématique, la physique et la logique sont les techno-sciences de la raison, puisque leur usage de la raison ne sert qu'à des fins accidentelles ou empiriques. Mais la philosophie, au contraire, transcende la technique. Elle n'obéit qu'aux fins essentielles de la raison humaine. Par conséquent, le philosophe seul mérite du nom de "législateur de la raison humaine». Ainsi la technicité s'écarte de la philosophie proprement dite. Et son caractère originel, non-technicisable assure une primauté de la philosophie réelle sur d'autres sciences, parce que la philosophie en réalité a le droit de systématiser des connaissances pour s'approcher de la philosophie arché-typique en tant que "système de toute connaissance philosophique». Elle fait d'un agrégat de connaissances un système selon l'essence de la raison humaine. Kant appelle cela «architectonique». Mais d'autres sciences en sont dépourvues. Quand elles rendent cohérentes des 
connaissances, elles n'obéissent qu'aux buts empiriques ou accidentels. Cette unité n'est que "technique». Bien entendu, l'«architectonique» est une technique, qui se définit comme «art des systèmes ${ }^{12}$ ". Mais l' "architectonique" répond à l'injonction essentielle de la raison, quoique la "technique" relève de l'empirique ou de l'accidentel. Nous pouvons affirmer donc que l' "archi-tectonique" philosophique est une technique "originelle», c'est-à-dire une super-technique, ou même une technique au-delà d'elle-même ou une technique liée au nontechnicisable. On voit ici une hiérarchie au sein de la philosophie originelle, de la philosophie réelle et des autres sciences. Leur ordre se détermine suivant leur relation avec la technique et l'origine. Dans la mesure où une science s'éloigne de la technicité et s'approche de son origine, elle a plus de supériorité. La philosophie ne débouche donc sur son essence originelle que si elle dépasse la technique. D'où une réaffirmation du rapportaristotélicien de la philosophie à l'origine età la technique.

III) Parallèlement au primat de son savoir, la philosophie joue un rôle principal dans l'institution universitaire. Dans $L e$ conflit des Facultés, Kant distingue la faculté inférieure de la faculté supérieure. La faculté inférieure est nommée faculté de philosophie. Elle contient deux départements : celui de "connaissance historique" (l'histoire, la géographie, la philologie scientifique, les humanités, les sciences de la nature) et celui des <<connaissances rationnelles pures >> (la mathématique pure, la philosophie pure, la métaphysique de la nature et des mœurs). Enfin, Kant va jusqu'à dire qu'elle «s'étend à toutes les parties du savoir humain (par conséquent, aussi, aux facultés supérieures) ${ }^{13}{ }^{\prime}$. La faculté de philosophie traite ainsi d'un vaste domaine - la totalité des sciences. Mais à la différence des facultés supérieures enseignant leurs spécialités et leurs techniques spécialisées, cette faculté fait de toutes ses connaissances non pas "son contenu, mais l'objet de son

12. Kant, op. cit., B860, p.1384.

13. Id., "Le Conflit des Facultés", in Oeuvres philosophiques III, tr. fr. par Alain Renaut, Gallimard, pléiade, 1986, p.826-827. 
examen et de sa critique en vue du profit des sciences ${ }^{14}$ ". Voici son trait fondamental. De même que les Critiques de Kant donnent une base à la construction de la métaphysique, la faculté de philosophie est un lieu basique où toutes les sciences sont examinées avant que l'on entre dans l'étude de leur contenu. Elle fait un travail préparatoire aux études spéciales futures : "simplement produire la vérité pour le bien de chaque science et la mettre à la libre disposition des facultés supérieures ${ }^{15}$ ". Un autre caractère de cette faculté est l'indépendance. Kant écrit : "La faculté de philosophie devra donc, parce qu'elle doit garantir la vérité des enseignements qu'elle doit accueillir ou même simplement accorder, être en tant que telle considérée comme libre et soumise uniquement à la législation de la raison, non à celle du gouvernement ${ }^{16}{ }^{\prime}$. Elle est entièrement autonome en raison de son droit de récuser toutes les interventions extérieures, notamment du gouvernement. Bien loin de dépendre du pouvoir étatique, elle ne répond qu'à l'exigence de la raison. Cette autonomie est en contraste avec l'hétéronomie des facultés supérieures, car elles relèvent intrinsèquement de l'intérêt du gouvernement. Dans Le conflit des facultés, on trouve trois facultés supérieures: faculté de théologie, faculté de droit et faculté de médecine. Quant à leur origine et à leur division, elles révèlent des facteurs empiriques, bien qu'elles ne soient pas nées par "hasard "17. La faculté de théologie a été créée pour que le gouvernement puisse "avoir la plus grande influence sur l'intimité des pensées et les volitions les plus secrètes de ses sujets ${ }^{18}$ ". La faculté de droit pour qu'il puisse «tenir leur[=les sujets] comportement extérieur sous la bride des lois publiques ${ }^{19}$ ". La faculté de médecine, pour qu'il puisse "s'assurer l'existence d'un peuple fort et nombreux qu'il trouve à sa disposition pour ses desseins ${ }^{20}$ ".

14. Ibid., p.827.

15. Ibid., p. 826.

16. Ibid.

17. Ibid., p.818.

18. Ibid.

19. Ibid.

20. Ibid. 
Ces facultés supérieures se sont ainsi établies en réponse aux besoins empiriques du gouvernement et non pas à l'injonction de la raison.

Je remarque deux points.

(1) Cette division faite par Kant est plus ou moins fidèle à la tradition de l'institution universitaire de l'Occident. En général, il y a, au moyen âge, quatre facultés dans l'université possédant un studium generale complet. Par exemple, l'université de Paris. D'abord, on voit la faculté des lettres ou des arts libéraux, qui estune étape préparatoire où l'on donne des enseignements généraux ou fondamentaux, plus concrètement trivium (grammaire, logique, rhétorique) et quadrivium (musique, arithmétique, géométrie, astronomie). Les étudiants qui y ont obtenu le diplôme de maîtrise, sont autorisés à entrer dans les facultés supérieures : celle de théologie, celle de médecine et celle de droit. Elles se chargent des enseignements spéciaux. Au moyen âge, il existe déjà un dualisme entre l'enseignement général et l'enseignement spécial. La structure universitaire dont Kant parle ne reflète pas seulement la situation de son époque mais dépend aussi de la tradition du système pédagogique. La faculté de philosophie ne vit que dans la division traditionnelle de l'enseignement général et de l'enseignement spécial.

(2) La faculté inférieure, malgré son nom modeste, a une supériorité sur les facultés supérieures, car elle a le droit d'examiner toutes les connaissances des autres sciences et d'en savoir le fondement, indépendamment de toute contestation du gouvernement. Cette prépondérance de la faculté couronnée du nom de philosophie est doublée par la relation hiérarchique de la philosophie avec les sciences spéciales. De même que la philosophie fournit une unité "architectonique" aux connaissances, aux sciences, aux techno-sciences, la faculté de philosophie donne une vérité fondamentale aux sciences en vue de rendre possibles les études des facultés supérieures. Cela au nom de la raison. Parce que cette faculté est «soumise uniquement à la législation de la raison". On doit se rappeler que le philosophe idéal se nomme «législateur de la raison 
humaine" dans la Critique de la raison pure. La philosophie originelle est la législation de la raison. Par conséquent, si la faculté de philosophie peut exercer son pouvoir sur d'autres sciences, c'est qu'elle obéit à la législation de la raison, c'est-àdire à la philosophie originelle en tant qu'idée. Dans sa pensée de l'institution universitaire, Kant maintient la double face de la philosophie : philosophie-origine et philosophie-copie. La hiérarchie que l'on découvre dans la Critique de la raison pure apparaît également dans $L \theta$ conflit des facultés. L'ordre scientifique qui se détermine selon le degré de l'origine et de la technique se répète dans le système pédagogique. La primauté de la philosophie - super-technique ou technique nontechnicisable - sur d'autres techno-sciences s'assure, même dans les rapports des facultés, par l'idée philosophique archétypique non-technicisable. De là une structure indivisible de l'institution universitaire et de l'éminence philosophique ${ }^{21}$.

IV) Depuis la révolution industrielle, le développement de la technique est remarquable. Nous bénéficions de la société techno industrialisée : développement des moyens de communication (téléphone, télévision), celui des moyens de transport (voiture, train, avion), augmentation de la durée moyenne de vie, etc. Mais nous n'en sommes pas moins menacés par la guerre nucléaire et la destruction de l'environnement. De surcroît, au fur et à mesure que la structure de la société change, la pratique, la méthode et l'institution de l'éducation se modifient, se renouvellent ou se réorganisent. Nous considérons comme un bon exemple la création de l'institut universitaire de la technologie, qui répond immédiatement aux besoins du milieu industriel, et qui s'oppose à l'idée classique de l'université. Aujourd'hui même, les vagues de la techno industrialisation ne

21. Je dois avouer que je me suis référé aux études de Jacques Derrida sur l'enseignement philosophique : "Chaire vacante: censure, maîtrise et magistralité" et "Les pupilles de l'université. Le principe de raison et l'idée de l'Université" (Jacques Derrida, Du Droit à la Philosophie, Galilée, 1990). Surtout la première étude m'a appris beaucoup de choses sur le rapport de "L'architechtonique de la ralson pure" de la Critique de la Raison pure et du Conflit des Facultés. J'al fait une lecture de ce rapport selon le point de vue de la technique. 
cessent d'exiger del'université une réforme dontl'aspect principal est une solidarité de l'université et de l'entreprise ou une subordination du système éducatif à l'industrialisation. Dans le contexte français, on remarque le projet Devaquet de 1985, qui détermine une multiplication des diplômes universitaires sur le plan régional pour aider les étudiants à intégrer le marché du travail; ou le plan Jospin, qui propose la réduction du temps scolaire des langues classiques en raison de leur peu de valeur pragmatique et augmente le nombre de stages pour la formation professionnelle $e^{22}$. Il en va de même pour le contexte japonais où l'on aborde la réforme universitaire sous le décret de 1991 du ministère de l'éducation nationale. II a pour principe de créer une communauté où l'université et la société industrialisée partagentle savoir. Le savoir spécial-technique - par exemple, résultats des études fondamentales - s'ouvre à la société, au public, auxentreprises. C'est pourquoi les salariés de l'entreprise peuvent assister aux cours de maîtrise et de doctorat et obtenir leurs diplômes, quoique ces cours soient à l'origine créés pour la formation des chercheurs-spécialistes. II ne faut pas oublier néanmoins que cette ouverture fait intervenir l'intérêt de l'entreprise dans l'université. En outre, cette réforme transforme la configuration des facultés, car le nouveau décret du ministère de l'éducation nationale élimine la distinction expresse entre l'enseignement général et l'enseignement spécial et n'impose à chaque université aucune obligation pour ces enseignements. Si bien que la division de la faculté inférieure et de la faculté supérieure, telle que Kant l'a mentionnée dans Le conflit des facultés, perd sa raison légale dans le nouveau système éducatif, car elle correspond à la dichotomie de l'enseignement général et de l'enseignement spécial. Certes, les étudiants, avant la réforme, étaient obligés d'assister aux cours de la faculté des arts libéraux (c'est-à-dire de la philosophie à l'époque de Kant) ou d'une organisation similaire pour mieux s'adapter au savoir spécial de la faculté supérieure. L'idée universitaire de Kant

22. Voir Jacques Minot, Histoire des Universités françaises, PUF, Que sais-je? 1991. Surtout ch.II et III. 
était vivante. Mais aujourd'hui où la faculté des arts libéraux (ou de philosophie) perd sa valeur légale d'existence, elle commence à s'abolir ou à devenir une autre faculté chargée de l'enseignement spécial dans quelques universités: Université de Kyoto et Université de Kobe. II n'y reste que des facultés concernant les techno-sciences spécialisées ${ }^{23}$. Nous comprenons déjà la valeur symbolique de cet événement. L'abolition des facultés des arts libéraux (ou de philosophie) nous conduit à saisir que la philosophie en tant que fondement universel des sciences ou des techno-sciences n'a plus de sens. Elle représente aussi une destruction de la supériorité de la philosophie sur d'autres sciences, c'est-à-dire celle du savoir originel super-technique ou non-technicisable sur le savoir technique. On commence déjà à mettre en cause autant la tradition aristotélicienne que la philosophie "architectonique» de Kant et son royaume de la faculté de philosophie.

V) Qu'est-ce qui reste de la philosophie dans la société techno-industrialisée? Quel rôle doit-elle y jouer? Doit-elle croire naïvement à son éminence sur la technique ou à sa capacité de fonder toutes les techno-sciences? Ou bien faut il qu'elle devienne une techno-science spéciale de même que la faculté de philosophie se change en une autre faculté spéciale? En effet, la section de philosophie dans la faculté des lettres s'occupe, aujourd'hui, de la formation des spécialistes de la philosophie ou des chercheurs de l'histoire de la philosophie. Sera-t-il possible de considérer l'avenir de la philosophie en dehors de sa fin?

II me semble que l'éclatement de la faculté de philosophie ne suggère pas la fin de la philosophie mais son «tournant». Ce qui est mis en question avec l'esprit actuel de la réforme, c'est la philosophie en tant que fondement universel des technosciences, qui dépend toujours d'une primauté de la connaissance philosophique originelle, super-technique ou non-technicisable surla connaissance technique. Le renouvellement de l'institution universitaire annonce que cette hiérarchie aristotélicienne ne 
fonctionne pas bien dans notre société contemporaine. Doit-on l'inverser pour contribuer à l'idéologie de la technoindustrialisation? Non. La base sur laquelle elle repose est la même que le fondamentalisme philosophique. Ce qui importe, c'est de remettre en question la structure de ce dualisme hiérarchique. II nous faudra réfléchir, dans cette perspective, au rapport de la philosophie et de la technique. A cette fin, je réexaminerai la relation du non-technicisable et de la technique et celle de l'origine et du dérivé - par exemple la copie - qui ont longtemps appuyé l'hégémonie de la philosophie. Ne sera$t$-il pas possible de penser ces relations autrement que de façon traditionnelle, comme celle de Kant?

Je considère le cas des beaux-arts. Ceux-ci sont des techniques, comme le mot art (Kunst) l'indique. Ils appartiennent étymologiquement à la $\tau \varepsilon \chi v \eta$. Mais ils sont aussi des nontechniques, car ils se distinguent des travaux du cordonnier ou del'horloger. Cette sorte d'activité que l'on entend ordinairement par le mot $\tau \varepsilon \chi \vee \eta$ ne se nomme pas beaux-arts. Ils sont un lieu exemplaire où s'annonce une relation ambiguë de la technique et du non-technicisable. De plus les beaux-arts se changent en s'exposant aux vagues de l'essor industriel. Nous voyons aujourd'hui les beaux-arts qui n'ont pas existé à l'époque de Kant. Par exemple, photographie et cinématographie. Leur changement n'exige-t-il alors pas de nous une modification de la perspective esthétique? Cela est plus ou moins parallèle à la réorganisation des facultés universitaires. Le renouvellement continuel techno-scientifique est à l'origine de la réforme universitaire, de même qu'il ébranle la pensée des beaux-arts. Pour repérer un changement concernant la technique, commençons par analyser le point de vue esthétique de Kant. Dans la Critique de la faculté de juger, ce philosophe dit: «les beaux arts sont les arts du génie ${ }^{24}$ ». Et "le génie est la disposition innée de l'esprit (ingenium) par le truchement de laquelle la nature donne à l'art ses règles ${ }^{25}$ ". Ce qui m'intéresse,

24. Emmanuel Kant, "Critique de la Faculté de juger", in Oeuvres philosophiques III, tr. fr. par A. J.-L. Delamarre, Gallimard, pléiade, 1985, p.1089.

25. Ibid. 
c'est que la nature constitue une partie essentielle des beauxarts. Bien que les beaux-arts soient des techniques, ils sont aussi des dons de la nature. Ils appartiennentà la fois à la $\tau \varepsilon \chi \nu \eta$

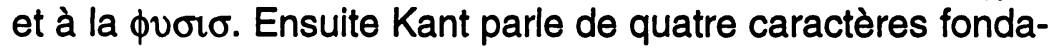
mentaux du génie. Le premier est «l'originalité" : "le génie» "est un talent, qui consiste à produire ce pour quoi on ne saurait donner aucune règle déterminée ${ }^{26}$ ". II produit des œuvres originales. Si ses ouvrages restent dans le cadre des règles déterminées, il ne sera pas vraiment génie. Deuxièmement, Kant montre l'exemplarité de l'œuvre du génie : «(...) dans la mesure où l'absurde peut être lui aussi original, les productions du génie doivent être également des modèles c'est-à-dire exemplaires $(. . .)^{27}$ ". Bien que ses $œ u v r e s$ ne soient pas produites par imitation, elles servent de modèles aux imitateurs. Elles deviennent les objets de l'imitation. La troisième propriété du génie est son incapacité d'expliquer scientifiquement: " [le génie] n'est pas lui-même en mesure de décrire ou de montrer scientifiquement comment il crée ses productions $[. . .]^{28}$ ". Son invention artistique est irréductible aux règles scientifiques, techniques, scolaires. II sera impossible d'en déduire des lois scientifiques. Quatrièmement Kant dit : «[...] à travers le génie la nature prescrit ses règles non à la science, mais à l'art [... ${ }^{29}$ ". Le génie est limité au domaine des beaux-arts. Même la découvert de Newton n'a rien à voir avec lui. De ces quatre définitions du génie, je tire deux conséquences.

(1) Dans la thèse kantienne, on voit une distinction tranchante de l'origine et de la copie. L'œuvre d'art est, en deux sens, une origine. Elle est originale dans la mesure où elle n'est pas une imitation. Elle est aussi originelle parce qu'elle est un objet de copie. Pour autant que l'œuvre d'art dépend du génie, elle constitue une origine : «[...] le génie est totalement opposé à l'esprit d'imitation ${ }^{30}$ ". 
(2) L'opposition de l'origine et de la copie correspond à celle de la $\phi v \sigma i \sigma$ et de la $\tau \varepsilon \chi v \eta$. Les produits de la nature sont toujours originaux et originels. Ils ne sont jamais engendrés par l'imitation. Même leur producteur ne peut les refaire en posant une règle mécanique de leur production. Comme ils ne sont pas répétables, leur singularité est absolue. Les activités scientifiques, au contraire, dépendent plus ou moins de l'imitation, parce qu'elles sont répétables, régularisables ou technicisables. Une fois découvertes, les règles scientifiques rendent possibles les imitations mécaniques et les répétitions scolaires. Autant le découvreur que les imitateurs peuvent répéter suivant les règles. Kant dit: «Dans le domaine scientifique, il n'y a donc que des différences de degré entre les plus inventeurs et les plus laborieux imitateurs etépigones ${ }^{31}{ }^{\prime}$. Cela diffère essentiellement de la singularité absolue du génie, qui rompt totalement avec l'imitation, la répétition, la technique. Enfin, l'œuvre d'art, en tant que don de la nature, garde son caractère original et originel, alors que la science appartient, en raison de sa «régularisabilité», plus ou moins à la copie, au mécanisme, au scolaire. Le dualisme de l'origine et de la copie est ainsi intrinsèque à celui de la $\phi v \sigma i \sigma$ et de la $\tau \varepsilon \chi v \eta$.

Certainement, le don de la nature est une origine des beauxarts. Est-ce à dire que l'œuvre d'art soit étrangère à l'apprentissage mécanique? Tout en soulignant le génie artistique, Kant n'oublie pas de dire qu'il requiert la technique scolaire : "Bien que les arts mécaniques soient très différents des beaux-arts les premiers en tant qu'arts reposant simplement surl'application et l'apprentissage, les seconds en tant qu'arts du génie - tous les beaux-arts sans exception admettent pour condition essentielle de l'art un élément d'ordre mécanique qui peut être appréhendé et appliqué selon des règles, donc recèlent néanmoins un élément scolaire ${ }^{32}$.. II faudra que le poète apprenne des règles prosodiques ou que le peintre connaisse des techniques du pinceau. L'exécution des beaux-arts ne se passe pas de l'application de la technique assimilée dans 
l'apprentissage scolaire. Sans employer des règles mécaniques, personne ne saura produire l'œuvre d'art. Par conséquent, Kant la divise en deux facteurs: "Le génie ne peut que procurer une riche matière aux productions des beaux-arts; le travail d'élaboration à partir de cette matière et la forme exigent un talent formé à une école [... $]^{33}$ ". Le génie constitue la matière des beaux-arts, alors que la technique scolaire est leur forme. Voilà une distinction de la matière originelle, originale et de la forme répétable, technicisable. En définitive, la réflexion kantienne sur le génie artistique repose sur une chaîne de couples opposés: origine/copie, $\phi v \sigma \sigma / \tau \varepsilon \chi \nu \eta$, matière/forme. II sera facile d'affirmer que ces distinctions sont fidèles à la tradition métaphysique. C'est par cette fidélité que la propriété d'origine du génie est toujours préservée de la technicisation, de la scolarisation, de la formalisation.

Mais le développement de la technique met en danger ces distinctions kantiennes. Ne devons-nous pas regarder ce que Kant n'a pas vu en raison des limites de l'époque et de la problématique métaphysique? Je m'en tiens à deux remarques.

(1) L'œuvre d'art se lie toujours à la possibilité de la reproduction. II y a des répliques que le peintre fait de l'œuvre originale; des copies que les disciples exécutent pour leur entraînement; des faux que l'on produit sous le nom de l'original. Dans la mesure où l'œuvre d'art est reproductible, elle est hantée par la relation de l'origine et de la copie. L'analyse kantienne du génie artistique fournit un bon exemple de la reproductibilité de l'œuvre d'art, en ce que le génie original crée l'œuvre originale ou originelle à l'opposé des copies ou des imitations. L'originalité du génie est un critère implicite de distinction entre l'original et l'imitation. Or au XIX $\mathrm{X}^{\ominus}$ siècle les techniques de reproduction marquent un pas remarquable de développement avec la lithographie et la photographie. Leur invention permet de produire une grande quantité de reproductions et de les jeter dans le marché du capitalisme. 
Aujourd'hui la reproduction joue un rôle important dans notre vie. Ce n'est pas seulement parce qu'elle laisse beaucoup de gens jouir de l'œuvre d'art, mais aussi parce qu'elle nous donne une autre perception. Par exemple, la reproduction photographique nous conduit, par un élargissement partiel du détail de l'œuvre originale, à voir ce qui échappe à la perception de la nature. À mesure que sa technique se développe, la reproduction acquiert une raison d'être indépendante de l'original. Je souligne, en outre, le plus fortement, que ce développement remet en question le rapport de l'original et de la copie. Dans L'œuvre d'art à l'époque de sa reproduction mécanisée, Benjamin montre un exemple intéressant: «Un cliché photographique, par exemple, permet le tirage de quantité d'épreuves: en demander l'épreuve authentique serait absurde ${ }^{34}$ ". Dans l'art photographique, il ne s'agit plus de la distinction de l'original et de la copie ni celle de l'authentique et du faux. Ces critères sont difficilement applicables à cet art. Un cliché peut produire ou reproduire un nombre infini d'originaux. La production est déjà une reproduction. II en est de même pour l'art cinématographique. Un film peut se produire ou se reproduire indéfiniment. Par conséquent, le même film peut passer simultanément dans beaucoup de cinémas. La reproductivité s'impose déjà dans la production. Benjamin dit : «Pour les films, la reproductivité ne dépend pas, comme pour les créations littéraires et picturales, d'une condition extérieure à leur diffusion massive. La reproductivité mécanisée des films est inhérente à la technique même de leur production. Cette technique, non seulement permet la diffusion massive de la manière la plus immédiate, mais la détermine bien plutôt ${ }^{35}$ ". Lorsque l'on produit un film, cette production a une structure de reproduction. La reproductivité, loin d'être accidentelle, est intrinsèque à la technique de la production. En somme, l'art photographique et l'art cinématographique débordent l'opposition traditionnelle de l'original et de la copie ou celle de l'authentique et du faux. Pour

34. Walter Benjamin, "L'œuvre d'art à l'Epoque de sa Reproduction mécanisée", in Ecrits français, tr. fr. par J.-M. Monnoyer, p.146.

35. Ibid., note. 
eux, l'œuvre originale est déjà une copie, l'imitation est déjà un original. Nous ne pouvons y considérer l'original que comme copie, la copie que comme original. Ces deux arts qui n'ont pas existé dans l'époque de Kant exigent de notre part une remise en question du dualisme classique. Kant a pensé la reproduction à partir de la production, si bien qu'il l'a limitée à l'imitation mécanique, scolaire ou scientifique. La reproduction a donc moins de valeur que la production du génie. Le non-technicisable l'emporte sur le technicisable. Mais aujourd'hui où la distinction de l'original et de la copie est remise en question, ne faut-il pas penser ce problème autrement que Kant? Au moins quant à la photographie et à la cinématographie, on doit penser la production et la reproduction à partir d'une reproductibilité, plus précisément d'une autre reproductibilité qui est à la fois originale et copie, mais n'est ni originale ni copie.

(2) Ne doit-on pas élargir la pensée de reproductibilité de Benjamin? Cette autre reproductibilité ne se voit-t-elle pas dans toutes sortes d'œuvres d'art? Ce qu'il nous faut aujourd'hui, ce n'est pas de maintenir la hiérarchie de l'origine et de la copie, mais plutôt de la repenser, dans tous les beaux-arts, à partir de cette reproductibilité. Ne peut-on pas trouver cette dernière soit dans l'œuvre originale du maître, soit dans l'imitation du disciple, soit dans le faux du tricheur? Dans cette perspective, je ferai, d'abord, état de l'analyse de Freud sur la production artistique de Léonard de Vinci. L'étude psychanalytique contribue, me semble-t-il, à la pensée de la reproductibilité dans l'exécution artistique. Freud écrit: "[Léonard de Vinci] rencontre la femme qui éveille en lui le souvenir du sourire heureux et sensiblement ravi de sa mère, et sous l'influence de ce réveil il retrouve l'impulsion qui l'avait guidé au début de ses essais artistiques, lorsqu'il créait des images de femmes souriantes. II peint La Joconde, la Sainte Anne en tierce et la série des tableaux mystérieux caractérisés par le sourire énigmatique. À l'aide de ces notions érotiques les plus archaïques, il célèbre son triomphe sur l'inhibition, encore une fois surmontée dans son art ${ }^{36}$ ". II ne

36. Sigmund Freud, Un souvenir d'enfance de Léonard de Vincl, tr. fr. par J. Altounian, A. et O. Bourguignon, P. Cotet et A. Rauzy, Gallimard, p.174. 
s'agit plus de savoir si Léonard prend conscience ou non du souvenir de son enfance dans l'exécution artistique. Ce qui importe, c'est que s'y répète son rapport infantile avec la mère. La Joconde, la Saint Anne en tierce et la série des tableaux témoignent de cette répétition inconsciente. Sa production n'est pas une origine, mais un recommencement. La production est déjà une reproduction. Cela ébranle la thèse kantienne du génie artistique, fondée sur la séparation tranchante de la création et de l'imitation, parce que l'artiste imite ou répète, dans son exécution, "quelque chose" de refoulé, qu'il s'en aperçoive ou non. La production artistique n'est pas purement originelle, mais suppose une structure récurrente. Bien entendu, cette reproduction n'est pas une réitération simple, car, lorsque "quelque chose" de refoulé se manifeste, il implique un déguisement, une transformation ou un déplacement. II ne se répète qu'en se différenciant. Par ailleurs, la reproduction n'est pas limitée au rapport de Léonard avec sa mère, quoique l'analyse freudienne reste centrée sur lui. Je me propose donc d'ouvrir sa recherche de Léonard vers ses autres ouvrages. La reproduction du refoulé n'est pas singulière, mais plurielle. $\mathrm{Si}$ l'on fait une lecture de la théorie postérieure de Freud, il faudra tenir compte d'une hétérogénéité de la reproduction. Le souvenir léonardien refoulé de sa mère dépend du principe du plaisir, mais dans L'inquiétante étrangeté., ce plaisir ne se détermine pas univoquement. II s'y agit du plaisir répétitif qui se caractérise par l'Un-heimlichkeit: à la fois étrangeté et familiarité. Le plaisir devient son contraire en même temps qu'il est lui-même. La reproduction d'un refoulé est ouvert à celle d'un indéterminable ou d'un indécidable du plaisir ou du déplaisir. Dans L'au-delà du principe du plaisir, Freud pense la répétition du déplaisir, c'està-dire «compulsion de la répétition» qui ne se plie pas au principe du plaisir. Elle lui fait postuler l'existence de «la pulsion de la mort» qui domine l'homme, plus fondamentalement que le principe du plaisir. On ne peut pas douter donc de la complexité du retour du refoulé. Cela dit, la reproductibilité inconsciente est un mouvement récursif ouvert à la pluralité et à l'ambiguïté. Il est ainsi évident que l'inconscient se rapporte étroitement à la 
reproductibilité. Mais je n'ai aucune intention de considérer l'inconscient ou individuel ou collectif comme absolu, car la reproductibilité ne trouve pas son origine absolue dans l'inconscient, mais s'ouvre déjà au dehors. Au-delà des problèmes de l'inconscient, la reproductibilité s'élargit à la problématique sémiologique, parce que ce qui se reproduit dans l'œuvre d'art, ce n'est pas seulement les refoulés inconscients de l'individu, mais encore d'autres facteurs. Par exemple, la femme qui éveille en Léonard le souvenir de la mère, les peintures qu'il a appréciées ou aimées, les idées ou thèmes artistiques communément répandues à son époque, etc. En somme, c'est ce qui lui adresse son signe ou à son conscient ou à son inconscient. Les refoulés inconscients sont aussi des signes "auto-affectifs". Les signes innombrables, se rapportant l'un à l'autre, se réinscrivent dans la production. Mais il faut remarquer un point : ce que j'entends par le mot "signe», ce n'est pas seulement le signe qui signifie dans la conscience ou l'inconscience, mais aussi «quelque chose" qui se signale dans l'oubli absolu mais se répète dans l'œuvre. Dans le signe, se soussigne un autre signe. Celui-ci se reproduit avec le signe signifiant, mais se dissimule dans la communication sémantique. Le signe garde son altérité. Pensons l'Être de Heidegger, qui reste insaisissable et oublié par la pensée métaphysique. On n'a pensé, au nom de l'être, que l'étant au cours de l'histoire de la métaphysique. L'Être ne cesse pas de se manifester en s'exposant à l'oubli absolu. Heidegger nous recommande de fixer notre attention sur le Wink de la parole : «[...] la parole est faire-signe (Wink) et non signe (Zeichen) au sens de la simple signification ${ }^{37}$ ". En même temps que la parole signifie, elle nous signale l'Etre au-delà de notre perception ou de notre compréhension sémantique, de façon que ce signal s'oublie dès sa réception. Cet autre signe est toujours déjà tout autre que le présent, le présent passé, le présent futur, et donc ouvert, au-delà de la mémoire vive, à l'immémorial et à l'à-venir. C'est pourquoi il continue, loin de se réduire au retour circulaire des

37. Martin Heidegger, "D'un Entretien de la Parole", in Acheminement vers la Parole, tr. fr. par J. Beaufret , W., Brokmeier et F. Fédier, Gallimard Tel, 1976, p.113. 
refoulés inconscients, de se reproduire en déjouant l'attente ou consciente ou inconsciente. Sa reproduction échappe toujours au présent et à la présence dans la communication du signe. Le signe soussigné de l'Être est ce que l'on n'attend que dans l'oubli absolu. Le signe implique ainsi un autre signe sans lequel il perdrait sa possibilité de reproduction. Les textes de Heidegger nous montrent exemplairement une duplicité sémiologique ${ }^{38}$. Pour autant que l'on pense la problématique du signe, il faudra donc prendre en considération cette différence "sémiologique". Or on voit reproduits dans le tableau de Léonard de nombreux signes qui constituent des strates hétérogènes diversifiées : consciente, inconsciente, extérieure, intérieure, sémantique, symbolique, sémiotique, etc. On pourra concevoir une image du "réseau" sur les rapports mutuels, multipliés, hétérogènes, fluctuants de ces signes. Mais il ne faut pas oublier qu'une infinité de signes ne se passent pas des autres signes reproductifs et absolument oubliés les rendant possibles. Le signe soussigné est très proche du signe, mais leur proximité ne se maintient que par un éloignement. Leur différence ne se réduit plus à celle de degré ni à celle entre un signe et un autre. Leur hétérogénéité est plus hétérogène que celle entre les strates des signes. S'il en est ainsi, il ne s'agira, dans le tableau de Léonard, plus d'une

38. Le signe soussignén'est pas restreintà l'Etre de Heidegger. À parler grossièrement, il est exploré, à leur façon, par Maurice Blanchot et Jacques Derrida. Pour rechercher à fond le signe de l'autre, il faudra non seulement se référer à ul'Être» de Heidegger, mais encore examiner ce que Blanchot entend par le "neutre», le "mourir", le "désastre», et ce que désignent les termes stratégiques de Derrida : "trace", "différance", "espacement", "hymen", "dissémination", etc. Ensuite, je tiens à noter que la négligence de la différence "sémiologique" par bien des sémiologues ne contribue qu'à l'affirmation nouvelle de la vieille métaphysique. En faisant une lecture des textes de Heidegger, Alexander Garcia Düttmann nous recommande de remarquer une différence du Zeichen et duWink dont l'oubli sera celui de l'altérité : "Le signe (Zeichen) est censé appartenir à la métaphysique que le "signe» (Wink) déborde. (-) Remarquer un Wink, c'est faire l'expérience de l'altérité."; "Le Zeichen serait donc un Wink oublié, l'oubli du langage qui, pour l'homme, est l'oubli de son essence". (Alexander Garcia Düttmann, La Parole donnée, Galilée, 1989, p.71-72.) À cette remarque, je voudrais ajouter cecl : dans l' "essence" de Wink, s'inscrit déjà structuellement un oubli. Ne sera-t-il pas impossible de penser le Wink sans l'oubli absolu? Le Wink ne continue pas de se répéter ou de se reproduire sans s'oublier. Sa reproductibilité requiert sans cesse son oubli. 
cohérence monolithique, mais de nombre de strates hétérogènes qui s'ouvrent à l'altérité : la reproductibilité hétérogène est doublée par la différence "sémiologique». Enfin, les reproductions doublement "sémiologiques" se marquent dans son tableau, que Léonard en prenne conscience ou non. Leurs effets n'apparaissent plus mécaniquement. Quelque effort qu'on fasse pour les réduire à un mécanisme, leur "mouvement» déborde à la fois le mécanisme sémiotique et le mécanisme psychique, caril implique ce qui échappe par nature au système du déchiffrement sémantique aussi bien qu' à la représentation et au registre de la mémoire. II se retire subrepticement du déterminisme psychanalytique et de la totalisation sémiologique. Je ne peux pas ne pas penser donc le «mouvement» irrégulier de la reproductibilité hétérogène dont un effet est l'œuvre d'art. Même si l'originalité du génie artistique existe comme le dit Kant, elle la présupposera. L'œuvre d'art n'est pas purement originale, mais déjà contaminée par les reproductions. Il en est de même pour l'imitation, qui ne se définit plus comme formelle, mécanique, scolaire, carl'imitation, par exemple, du refoulé, requiert à la fois la forme et la matière. Plutôt que de réduire l'imitation aux procédés formels, je préfère penser qu'elle est aussi un résultat des reproductions hétérogènes, doublement "sémiologiques". Autant la matière que la forme d'une œuvre d'art sont surdéterminées par elles, mais d'une autre façon que le "déterminisme" et le "surdéterminisme». Cela avec une réimpression, dans cette surdétemination, de ce qui l'excède. C'est pourquoi la reproductibilité précède la distinction de l'originalité et de l'imitation, ou celle de la matière et de la forme. Je peux dire la même chose à propos de l'opposition de la $\phi v \sigma i \sigma$ et de la $\tau \varepsilon \chi \vee \eta$, puisque la reproductibilité se produit non seulement dans la production artistique mais aussi dans d'autres activités. Ces reproductions se trouvent soit dans l'invention artistique, soit dans la pensée scientifique, soit dans l'activité névrotique, soit dans la vie quotidienne de n'importe qui. S'agissant de cette reproductibilité, le privilège de l'art en tant que don de la nature perd son sens. Elle agit "avant» la distinction de la $\tau \varepsilon \chi \vee \eta$ et de la $\phi v \sigma \tau \sigma$, ou celle du technicisable 
et du non-technicisable. Elle n'est ni technicisable ni nontechnicisable, mais à la fois technicisable et non-technicisable. Enfin, la pensée de la reproductibilité remet en question une chaîne d'oppositions conceptuelles origine/imitation, $\phi v \sigma r /$ $\tau \varepsilon \chi \vee \eta$, matière/forme, car la pensée métaphysique a longtemps opprimé la reproductibilité passant librement de l'un à l'autre des territoires opposés et rendant possibles ces séries d'oppositions. II faut donc examiner encore, à partir de la reproductibilité, ces dichotomies déjà figées et les valeurs hiérarchiques apportées par elles. Un exemple en est fourni par la relation du génie artistique et de l'imitateur. L'esthétique de Kant, qui a exclu l'imitation du concept de génie, appelle, avec un mépris, l'imitation totale singerie : "(...) cette imitation devient singerie lorsque l'élève imite tout, et même les malformations que le génie n'a pu faire autrement que tolérer $(. . .)^{39}$ ". Mais l'imitation à cent pour cent est-elle possible? Même si cet élève imite tout, il ne fait pas que copier mécaniquement l'œuvre du maître. Dans son imitation, se reproduisent autant les signes "extérieurement affectifs" ou "auto-affectifs" que les effets signaux de l'immémorial. Affecté par la femme, Léonard imite son enfance refoulée dans l'exécution artistique, de même que son imitateur, influencé de quelque façon par l'ouvrage du maître, répète ses refoulés dans son travail. En outre, se réinscrivent, dans l'œuvre de ce disciple, divers signes extérieurs ou intérieurs. Et aussi les signes non-signifiants tout autres. Indépendamment des intentions des artistes, l'imitation et l'œuvre fausse sont aussi singulières que l'original. Entre le génie artistique et l'imitateur, il n'y a pas de différences comme la "rupture». La pensée de la reproductibilité reconnaît à la hiérarchie métaphysique de l'original et de la copie la singularité et l'indépendance de chaque œuvre. Les reproductions produisent des effets singuliers dans leur «mouvement» hétérogène, doublé.

Des développements techno-scientifiques comme la photographie, la cinématographie, la psychanalyse ou la 
sémiologie nous conduisent à mettre en doute la distinction hiérarchique de l'origine et du dérivé (copie) ou celle du nontechnicisable et du technicisable. Mais il sera vain de renverser leur hiérarchie en répondant uniquement à l'exigence technoscientifique. Affirmées ou renversées institutionnellement, elles seront des effets de la reproductibilité hétérogène. Cette dernière traverse la frontière de l'origine et de la copie, et celle du nontechnicisable et du technicisable. Parce qu'elle est à la fois origine et copie, technicisable et non-technicisable, n'étant ni origine ni copie, ni non-technicisable ni technicisable. Avec la phase contemporaine de la techno-science, il est devenu possible de penser la reproductibilité, que la pensée métaphysique a dissimulée en prétendant le primat de la production sur la reproduction. Elle ne se limite pas aux beaux-arts - quoique je prisse l'exemple esthétique - mais s'étend à tous les domaines concernant la technique et le scientifique. Même la philosophie ne constitue pas une exception, car elle présuppose le dualisme de l'origine et de la copie ou celui du non-technicisable et du technicisable. La relation de l'origine et de la copie s'impose non seulement dans le platonisme, la doctrine de l'analogie de la théologie chrétienne, mais aussi dans la philosophiemoderne. Depuis la hiérarchie scientifique d'Aristote, le statut de la philosophie se détermine à la fois comme originel et comme non-technicisable au sens de la sur-technique ou de l'au-delà de la technique. Si la philosophie a l'avantage, chez Kant, de systématiser, de fonder toutes les connaissances, toutes les techno-sciences, c'est qu'elle est "architectonique», technique originelle ou technique non-technicisable, et qu'elle seule obéit au commandement de la raison, de son idée originelle, jamais technicisable. Ce primat de la philosophie se trouve également dans la configuration des facultés universitaires. La faculté de philosophie acquiert une autonomie indépendante autant du gouvernement que des facultés spéciales. Mais à mesure du développement techno-scientifique, la faculté de philosophie commence, aujourd'hui, à perdre sa signification, comme le représente l'éclatement de la faculté des arts libéraux au Japon. La hiérarchie scientifique depuis 
Aristote dontle sommet est la philosophie fonctionne difficilement dans la société actuelle. À un doute s'exposent, aussi, la dichotomie de l'origine et du dérivé (copie), et celle du nontechnicisable et du technicisable, qui ont étayé l'hégémonie scientifique de la philosophie. Dans cette situation, ne doit-on pas penser le rapport de la philosophie et des techno-sciences à partir de la reproductibilité ni purement originelle ni purement copieuse? Même la pensée philosophique ne sera-t-elle pas un effet de la reproductibilité hétérogène, comme le sont le technicisable, la technique ou le scientifique? Dans une technoscience, des philosophèmes s'inscrivent déjà, se reproduisent, de même que des facteurs de cette techno-science se répètent dans la philosophie. Les facteurs de chaque science sont déjà disséminés, répétés, communiqués avec et au-delà de la barrière scientifique et indépendamment de la conscience du chercheur. "Avant" que se déterminent les frontières et la hiérarchie des sciences ou que s'établissent les relations de l'origine et du dérivé (copie), il y a déjà une structure reproductive. En l'occurrence, nous ne devrons pas oublier de tenir compte de la reproductibilité tout autre, toujours déjà inscrite et oubliée dans diverses reproductions. Sinon notre essai retomberait dans les pièges du "déterminisme», du «totalisme-totalitalisme» ou du «mouvement circulaire» propres à la vieille métaphysique. Nous voyons déjà sa réhabilitation dans l'admiration naïve de la techno-science - par exemple la psychanalyse ou la sémiologie. Ce que l'on nous demande, ce n'est plus d'affirmer la distinction de l'origine et du dérivé (copie), celle du nontechnicisable et du technicisable, les frontières technoscientifiques, la hiérarchie philocentrique, non plus de déclarer leur annulation totale, non plus de renverser leur ordre hiérarchique au profit des techno-sciences mais de les examiner sous l'angle de la reproductibilité doublée. Ce point de vue ouvrira la porte aux diverses couches du savoir. La réorganisation universitaire ne doit-elle pas commencer par la pensée de la reproductibilité?

Takuji Iwano 